\title{
QUASAR EMISSION - LINE STRENGTHS
}

\author{
Richard Green, Patrick Osmer, and Alain Porter \\ Kitt Peak National Observatory \\ National Optical Astronomy Observatories
}

Keywords - Emission Lines

The strongest lines have been measured in 73 quasar spectra from the archives of the International U1traviolet Explorer Satellite. The optically bright quasars observed with IUE we re typically discovered by powerful radio emission or ultraviolet excess. They therefore should not be biased directly by observational selection with respect to ultraviolet line strength.

Similar measurements have been obtained from a new database of uniformly observed high-redshift quasars. A sample of 77 objects with $z>3$ includes most high-z quasars in the Hewitt-Burbidge Catalog; they were discovered primarily by slitless spectroscopy, while some were radio source identifications. A comparison sample of 36 quasars with z 2 was also observed. This large combined sample can be used to evaluate the selection efficiency of slitless spectroscopic techniques and to test for correlations of line strengths and continuum power, such as the Baldwin effect.

Histograms of rest equivalent widths for Lya and CIV show a shift of the median to lower values with redshift. The actual effect may be even more pronounced, since the photographic slitless spectroscopic surveys may be biased toward stronger lines.

The two upper plots show the significant anticorrelation of emission-1ine to continuum ratio with continuum luminosity, the Baldwin effect. The effect is more pronounced in CIV than in Ly $\alpha$, although it is present for both features. The rest "equivalent width" of CIV is fitted by

$$
\mathrm{W}_{0}(\mathrm{CIV})=65\left(\mathrm{~L}_{\nu}(1450) / 10^{30}\right)^{-0.28} \AA
$$

(for $H_{0}=50$ and $\Omega=1$ ). In contrast

$$
\mathrm{W}_{0}(\operatorname{Ly} \alpha)=102\left(\mathrm{~L}_{V}(1450) / 10^{30}\right)^{-0.14} A
$$

Note the difference of a factor of 2 in the exponents. These results are in good agreement with those of Kinney et al. (1987, Ap.J., 314, 145), especially when the difference in assumed $\Omega$ is taken into account. 
An explanation for the Baldwin effect was given by Mushotzky and Ferland (1984, Ap.J., 278, 558) as a luminosity dependence of the ionization parameter, $U$, the ratio of the ionizing photon density to the cloud electron density. The plot of line ratio vs. luminosity shows diagnostics that are sensitive to the ionization parameter. The fitted regressions show

corresponding to

$$
\left.\operatorname{Ly} \alpha / \operatorname{CIV} \propto \mathrm{L}_{\nu}-0.19 \text { and } \operatorname{CIII}\right] / \mathrm{CIV} \propto \mathrm{L}_{\nu}{ }^{0.20} \text {, }
$$

$\mathrm{U} \propto \mathrm{L}_{\nu}-0.19$ for $(\mathrm{Ly} \alpha / \mathrm{CIV})$, and $\mathrm{U \propto L} \mathrm{L}_{\nu}^{-0.23}$ for (CIII]/CIV).

The latter ratio is density dependent, while the former is not; CIII] is also the poorest quality measurement of the data set. If the small difference turns out to be real, it could reflect a weak dependence of cloud density on luminosity. Further evidence for this trend can be seen in the ratio-ratio plot in comparing the location of the objects relative to a mogel locus for varying ionization parameter with constant density of $10^{1} \mathrm{~cm}^{-3}$.
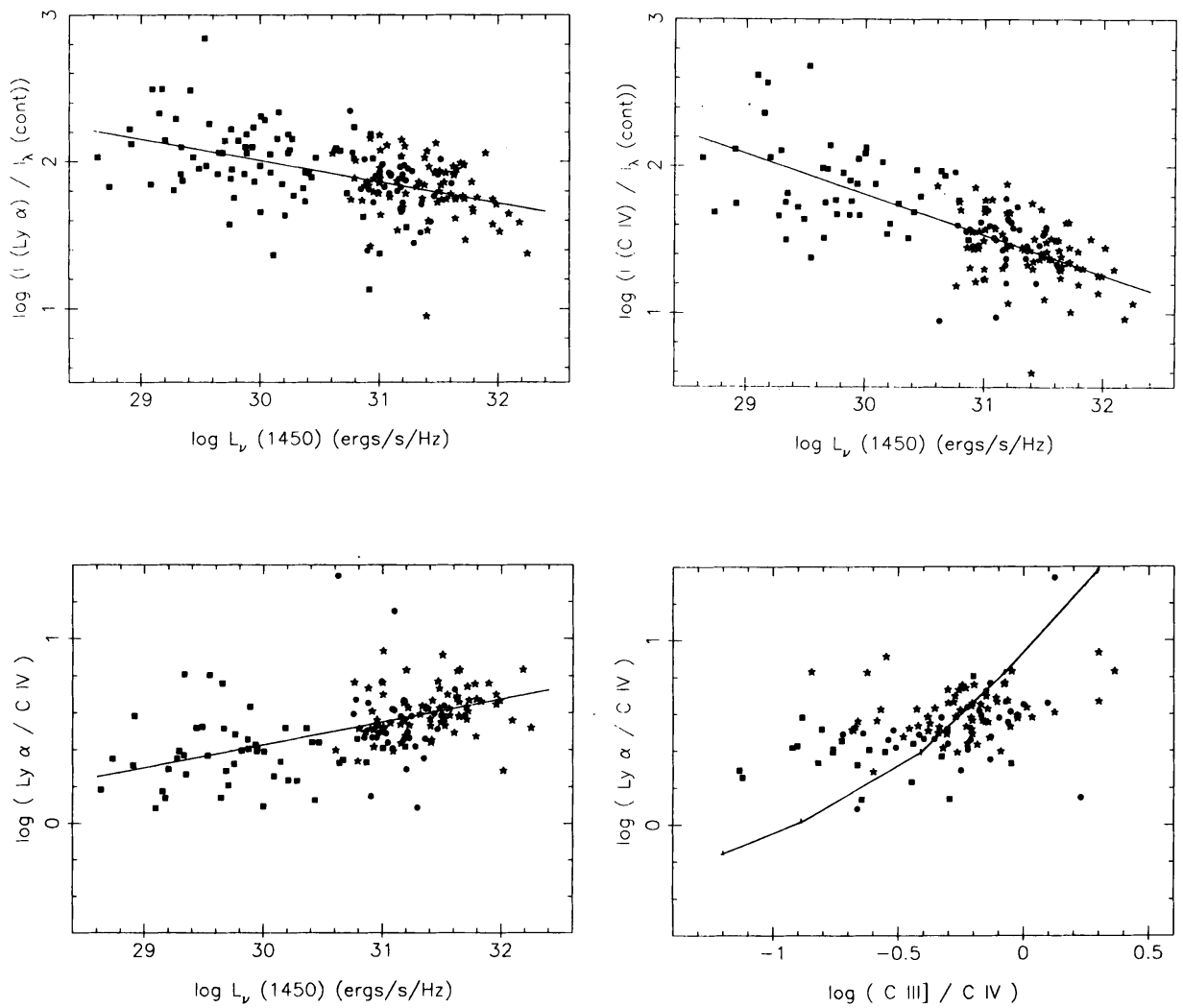